\title{
MED29, A Subunit of the Mediator Complex, Possesses Oncogenic Characteristics in Ovarian Cancer
}

\section{Haifang Wu}

Nanchang University Second Affiliated Hospital

\section{Ling Chen}

Nanchang University Second Affiliated Hospital

\section{Yiguo Wu}

Nanchang University Second Affiliated Hospital

Weiming Lou

Nanchang University

Penghui Nie

Nanchang University

\section{Xiaoli Tang}

Nanchang University Medical College: Medical College of Nanchang University

Fen Fu ( $\nabla$ fu_fen@163.com )

Nanchang University Second Affiliated Hospital

\section{Research Article}

Keywords: Mediator complex, Mediator complex subunit 29, ovarian cancer

Posted Date: August 19th, 2021

DOI: https://doi.org/10.21203/rs.3.rs-352662/v2

License: (9) This work is licensed under a Creative Commons Attribution 4.0 International License. Read Full License 


\section{Abstract}

Background: Somatic copy number alteration (SCNA) usually accompanies the appearance of tumours; one common example is the 19q13.2 region amplification (AMP), mediator complex subunit 29 (MED29) were an amplified effector gene within this region. In this study, the role and molecular mechanism of MED29 in ovarian cancer (OvCa), one of the three major tumours in gynaecology, were discussed.

Results: According to the transcriptome and survival data from the TCGA database, 19q13.2 AMP corresponded with a worse prognosis of OvCa patients $(P=0.0253)$, and individuals with 19q13.2 AMP showed

increased levels of MED29 mRNA. From the GSE29450 dataset, we found that the gene expression of MED29 was significantly upregulated in OvCa and overexpression of MED29 in OvCa was related to shorter survival. Additionally, pathway analysis based on RNA-seq data indicated that MED29 could activate the expression of genes involved in cell proliferation. Moreover, knockdown of MED29 obvious inhibited the proliferation, invasion and migration of OVCAR3 and A2780 cells and arrested the cell cycle at the $\mathrm{G} 2 / \mathrm{M}$ transition in vitro.

Conclusion: MED29 could be used as a potential therapeutic target for OvCa treatment.

\section{Introduction}

Ovarian cancer (OvCa) is one of the three major malignant reproductive system tumours in women, with a high degree of malignancy and a high mortality rate [1, 2]. Epithelial ovarian cancer (EOC) is the most common type of OvCa, and $70 \%$ of patients with EOC have high-grade serous ovarian cancer (HGSC) at the time of diagnosis [3]. As the life expectancy of humans increases, the number of diagnosed OvCa cases each year is also increasing [4]. The 5-year survival rate of patients receiving standard treatment (surgery plus combined paclitaxel and platinum-based drug regimen) is $31 \%$, and $5 \%$ of OvCa patients will exhibit drug resistance within half a year after treatment. The high recurrence rate of OvCa is related to cancer cell metastasis and chemotherapy resistance [3]. Therefore, there is an urgent need to find new treatment options for OvCa.

Genome mutations or aberrations acquired in normal cells and specific tissues are usually followed by the appearance of tumours [5]. Somatic copy number alteration (SCNA) is a more critical form of somatic mutation [6]. The tumour genome usually exhibits copy number variation (CNV) in somatic cells during the process of carcinogenesis, and the amplification of oncogenes or the deletion of tumour suppressor genes is usually pathogenic because the expression level of genes is highly correlated with copy number [7]. In fact, many cancer-related genes have been determined to be affected by SCNAs [8]. Some studies have shown that CNAs are a marker of cancer cells and contribute to carcinogenesis $[9,10]$.

The Cancer Genome Atlas (TCGA) conducted a comprehensive genomics study on OvCa (especially HGSC) and found that the somatic mutation pattern in OvCa was unique and dominated by SCNAs [11, 
12]. The integrative analysis of HGSC indeed identified a large number of focal SCNAs in the genome, some of which contain known important effector genes (for example, CCNE1, MYC, PTEN, RB1) that might have an effect on the tumour phenotype [12]. This indicates that SCNAs can directly affect the cell phenotype by changing the expression of effector genes in the region, and further produce changes in the tumour phenotype. However, the functional genes involved in most SCNAs and their relationship with tumour phenotype remain unclear.

The $19 \mathrm{q} 13.2$ region amplification (AMP) is a common SCNA in HGSC $(P=2.25 \mathrm{e}-28)$. This region is located between 39,738 kbp and 40,174 kbp on chromosome 19, and spans $438 \mathrm{kbp}$. Studies have shown that 19q13.2 AMP worsens the prognosis of approximately $12 \%$ of HGSC patients [12]. Genomewide association analysis based on epithelial mucinous ovarian cancer (MOC) suggested that polymorphisms in the 19q13.2 region (rs688187) were highly correlated with the pathogenesis of MOC ( $P$ = 6.8 -13 ), and 19q13.2 AMP was also associated with undifferentiated and more aggressive OvCa [13]. These results suggested that there are important effector genes in the 19q13.2 region related to the occurrence and development of EOC, and it is still necessary to systematically identify the effector genes in this region and explore the molecular mechanism of this aggressive phenotype in OvCa.

Therefore, in this study, the role of MED29 (located in the 19q13.2 region) in OvCa was explored. Data from the TCGA and GEO databases revealed that 19q13.2 AMP was significantly associated with poor survival and OvCa patients with 19q13.2 AMP had increased levels of MED29 mRNA. Furthermore, increased MED29 expression has the greatest impact on the progression-free survival (PFS) of OvCa patients. By conducting a comprehensive data analysis, we evaluated the effects of MED29 expression on patient prognosis and verified its effect on cell proliferation, cell cycle progression, and migration and invasion abilities by constructing cytological models. Therefore, our purpose was to demonstrate that MED2 9 is a potential therapeutic target for OvCa, providing an innovative option for the treatment of OvCa patients.

\section{Material And Methods}

\subsection{Bioinformatic analysis of TCGA data and GEO data}

The TCGA visual online database cBioPortal (https://www.cbioportal.org/) was used to analyse the survival of patients with OvCa and 19q13.2 AMP, the genes contained within 19q13.2, and the change in MED29 expression after gene CNAs. The Gene Expression Omnibus (GEO) database (https://www.ncbi.nlm.nih.gov/geo/) is a national public database, including high-throughput gene expression and array- and sequence-based data [14]. In this study, qualified data were obtained from the GEO dataset GSE29450 (Affymetrix Human Genome U133 Plus 2.0 Array; 10 samples of OvCa and 10 samples of normal tissue), and then the data were pre-processed and analysed with R software. A boxplot was built to show the differential analysis of specific gene expression between OvCa tissues and non-tumour tissues. Kaplan-Meier Plotter (http://kmplot.com/analysis/) was used to certificate the relationship between the dysregulated genes and patient survival. The pathway mapping of candidate 
genes by Kyoto Encyclopedia of Genes and Genomes (KEGG) analysis (http://www.webgestalt.org/website.pathway) was also executed.

\subsection{TMA and IHC}

A tissue microarray (TMA) comprising 80 formalin-fixed paraffin-embedded primary OvCa tissue dots with corresponding clinical annotations was purchased from Taibsbio.com (Xi'an, China).

Immunohistochemistry $(\mathrm{IHC})$ was performed to detect MED29 protein expression in tumour and normal tissues in the microarray. The TMA was sent to Servicebio Company (Hunan, China) for immunohistochemical analysis. Quantitative evaluation and automatic scoring of immunohistochemical images were performed with ImageJ software. This method is based on the research of Varghese $\mathrm{F}$ et al. [15]. The score was evaluated into 4 grades: 1 (almost negative), 2 (weakly positive), 3 (positive), and 4 (strongly positive).

\subsection{Cell culture}

OVCAR3 cells (Cell Resource Center, Shanghai Academy of Biological Sciences, Chinese Academy of Sciences) were cultured in RPMI 1640 medium (Solarbio, Beijing, China) complemented with 20\% FBS (Gibco), penicillin $(100 \mathrm{U} / \mathrm{ml})$ and streptomycin $(100 \mathrm{ng} / \mathrm{ml})$. A2780 cells (Cell Resource Center, Shanghai Academy of Biological Sciences, Chinese Academy of Sciences) were cultured in RPMI 1640 medium (Solarbio) complemented with 10\% FBS (Gibco), penicillin $(100 \mathrm{U} / \mathrm{ml})$ and streptomycin $(100 \mathrm{ng} / \mathrm{ml})$. ES2 cells were cultured in DMEM (Gibco) complemented with 10\% FBS (Gibco), levofloxacin penicillin (100 $\mathrm{U} / \mathrm{ml})$ and streptomycin $(100 \mathrm{ng} / \mathrm{ml})$. All cells were incubated at $37^{\circ} \mathrm{C}$ in $5 \% \mathrm{CO}_{2}$.

\section{4 siRNA-mediated MED29 gene silencing}

Two MED29 gene-specific siRNAs (295 and 426) and negative control siRNA (NC) were obtained from GenePharma, Shanghai, China. The siRNA sequences targeting MED29 were as follows: siRNA-295MED29, 5'-CAGAACACUAACAUCGACATT-3'; siRNA-426-MED29, 5'-GUCACAGAGUUGUGACAGUTT-3'; and siRNA-NC-MED29, 5'-CAGAACACUAACAUCGACATT-3'. In all, $5 \mathrm{nM}$ siRNAs were transfected into 60\% confluent cells using Thermo Turbofect transfection reagents (Thermo Fisher Scientific, UAB) accordance with the manufacturer's protocol. Then the cells were incubated in $5 \% \mathrm{CO}_{2}$ at $37^{\circ} \mathrm{C}$ for $48 \mathrm{~h}$ until subsequent analysis.

\subsection{RT-PCR analysis}

Total RNA from OvCa cells was extracted with an AxyPrep Multisource Total RNA Miniprep Kit (Axygen Scientific, Union City, CA, USA) accordance with the standard protocol. cDNA (20 $\mu$ l) was synthesized from $1 \mu \mathrm{g}$ of total RNA by a Takara PrimeScript ${ }^{\text {TM }}$ RT reagent kit and gDNA Eraser (Cat\# RR047A, Lot\# AK2802). Quantitative real-time PCR (RT-qPCR) by TB GreenTM Premix Ex TaqTM II (TakaRa Code: DRR820A) was fulfilled following the standard protocol on a 7900HT Fast Real-time System (Applied Biosystems, Foster City, CA, USA). GAPDH was used as the reference gene. The relative gene expression levels were determined using the critical threshold (Ct) number and calculated through the $2^{-\triangle \Delta C t}$ method. 


\subsection{Western blot}

At $48 \mathrm{~h}$ following siRNA transfection, cells were lysed with RIPA buffer and centrifuged at $12000 \times \mathrm{g}$ for 10 min in order to collect total protein. A PierceTM BCA protein assay kit (Thermo Fisher Scientific, UAB) was used to determine the protein concentrations in each sample. In all, $25 \mu \mathrm{g}$ of total protein was separated via SDS-PAGE and then transferred to polyvinylidene fluoride membranes. After they were enclosed in Tris-buffered saline (TBS) with $5 \%$ non-fat milk for $1 \mathrm{~h}$ to saturate additional protein binding sites, the blots were incubated with the following primary antibodies: anti-Med29 (clone sc-502, 1:500 dilution, Santa Cruz Biotechnology, Santa Cruz, CA), anti-Fibronectin (1:1000, Proteintech, cat15613-1-AP), anti-Ecadherin (1:5000, Proteintech, cat20874-1-AP), anti-N-cadherin (1:2000, Proteintech, cat22018-1-AP), antiVimentin (1:2000, Proteintech, cat10366-AP), anti-cyclinD1 (1:5000, Proteintech, cat60186-1-lg), anticyclinE (1:1000, Proteintech, cat11935-1-AP), and anti-cyclinB1 (1:1000, Proteintech, cat55004-1-AP) antibodies at $4^{\circ} \mathrm{C}$ for $12 \mathrm{~h}$, then incubated with horseradish peroxidase-conjugated anti-mouse or antirabbit secondary IgGs at room temperature for $2 \mathrm{~h}$ or at $4^{\circ} \mathrm{C}$ for $4 \mathrm{~h}$. The proteins were visualized through using a BM chemiluminescence Western blotting kit (Roche Diagnostics $\mathrm{GmbH}$ ). To guarantee equal loading and accuracy for assessment of the changes in protein abundance, the protein levels were standardized to those of GAPDH as a housekeeping control.

\subsection{Colony formation assay}

At $48 \mathrm{~h}$ after siRNA transfection, cells were inoculated in 6-well tissue culture plates at a density of 50 cells $/ \mathrm{cm}^{2}$ and cultured for $14 \mathrm{~d}$, after which colonies were fixed with ethanol, stained with $2 \%$ crystal violet, washed with PBS to remove excess dye, and imaged on a scanner. To determine the quantitative changes in clonogenicity by counting the number of colonies with ImageJ software.

\subsection{EdU cell proliferation assay}

Using incorporation of 5-ethynyl-2'-deoxyuridine (EdU) with an EdU Cell Proliferation Assay Kit (RIBOBIO, Guangzhou, China, Cat\# C10310-3) to detect cell proliferation. At $48 \mathrm{~h}$ following siRNA transfection, $5 \times 10^{3}$ OVCAR3 and A2780 cells were plated in 24-well plates, and the cells were incubated in complete medium under standard conditions. Images viewed under a fluorescence microscope were obtained.

\subsection{CCK8 assay}

Using the CCK-8 kit (US EVERBRIGHT INC, China, Cat: C6005-500T) to detected cell proliferation. Cells were inoculated in 96-well plates in $100 \mu \mathrm{l}$ of medium at approximately 3000 cells per well, and three independent parallel experiments were established. After cells were incubated at $37^{\circ} \mathrm{C}$ in $5 \% \mathrm{CO}_{2}$ for $24 \mathrm{~h}$, $10 \mu$ of CCK 8 was added into the wells after an additional 1, 2, 3, 4, 5 and 6 days and cultured for $2 \mathrm{~h}$. The absorbance in each well at a wavelength of $450 \mathrm{~nm}$ was measured.

\subsection{Cell migration assay}

Serum-starved OVCAR3 cells ( $2 \times 10^{4}$ cells) in $200 \mu$ l of 1640 medium complemented with $5 \%$ FBS were plated into the upper chamber of prepared transwells; the lower chamber contained medium 
supplemented with $20 \%$ FBS. A2780 cells $\left(1 \times 10^{5}\right.$ cells) in $200 \mu$ of serum-free 1640 medium were plated into the upper chamber of prepared transwells; the lower chamber contained medium supplemented with $10 \% \mathrm{FBS}$. After plates were incubated in $5 \% \mathrm{CO}_{2}$ at $37^{\circ} \mathrm{C}$ for $24 \mathrm{~h}$, the migratory or invading cells in the lower chamber were fixed and stained with $2 \%$ crystal violet. Five representative fields of view of each membrane were selected, microscopy images by were captured, and the number of migrating cells or invasive cells was counted with ImageJ.

\subsection{Cell cycle analysis by flow cytometry}

Forty-eight hours after transfection with siRNA targeting MED29, cells were fixed and stained with propidium iodide (PI) $(50 \mu \mathrm{g} / \mathrm{ml}$, Sigma). The tests were executed in triplicate. To perform accurate cell cycle analysis, the cells subjected to siRNA transfection were centrifuged at $1500 \mathrm{rpm}$ for $5 \mathrm{~min}$ and afterwards resuspended in $500 \mu \mathrm{l}$ of PBS, and $1.5 \mathrm{ml}$ of $95 \%$ ethanol $\left(-20^{\circ} \mathrm{C}\right.$ precooled) was added to fix the cells at $-20^{\circ} \mathrm{C}$ for 10 minutes. The cells were vortexed before they were centrifuged at $1500 \mathrm{rpm}$ for 10 min, after which the supernatant was discarded and the cells were resuspended in $500 \mu$ of PBS for 10 min before subsequent treatment with RNase $A$ at $37^{\circ} \mathrm{C}$ for 10 minutes. Ultimately, $\mathrm{PI}(2 \mu \mathrm{g} / \mathrm{ml})$ was added to the cells for $15 \mathrm{~min}$ to stain the DNA, and then the BrdU test was performed. BrdU analysis and cell cycle distribution were executed with BD CellQuest Pro $^{\text {TM }}$ on a BD FACSCalibur flow cytometer (BD Biosciences, New Jersey, USA) through obtaining a minimum of $2 \times 10^{4}$ mononuclear cells.

\subsection{Statistical analyses}

Statistical analyses were performed using SPSS 22.0 and GraphPad Prism 5 software. Each experiment was independently repeated at least triplicate. All data are expressed as the means \pm standard deviation (means \pm SD). One-way analysis of variance (ANOVA) was conducted to compare multiple groups, whereas Student's t-test was used for comparisons between two groups. For all analyses, two-tailed $p$ values below 0.05 were considered significant and are indicated as follows: ${ }^{\star} p<0.05,{ }^{\star \star} p<0.01$.

\section{Results}

\subsection{Comprehensive analysis showed the most obvious change in MED29 expression in samples with 19q13.2 AMP}

In this study, using cBioPortal to analyse the OvCa data from the TCGA database, 19q13.2 AMP showed a significant correlation with poor survival in OvCa patients $(P=0.0253)$, as shown in Fig. 1A. The 19q13.2 region contains 22 known genes, 19 of which are differentially expressed; and AMP of MED29 has the greatest impact on PFS of patients (Fig. 1B). Further analysis indicated that 19q13.2 AMP increased the mRNA level of MED29 in the region (Fig. 1C). Upon analysis of the expression levels of MED29 in 20 samples from GSE29450, it was found that MED29 expression in the OvCa samples was higher than the normal samples $(P=0.0015)$ (Fig. 1D). 


\subsection{MED29 is highly expressed in OvCa and is related to poor prognosis of OVCa}

To evaluate the clinical correlation between MED29 and OvCa, we performed IHC staining assays of the protein in ovarian TMA, including 75 OvCa samples. The results confirmed that the protein level of MED29, which was negligible in normal ovarian epithelium, was significantly increased in OvCa (Fig. 2A), and the higher MED29 protein levels correlated with higher IHC scores (Fig. 2B). In addition, Kaplan-Meier Plotter was used to analyse the effect of MED29 on the prognosis of patients with OvCa. The results showed that MED29 expression was closely related to overall survival (OS) $(n=1657)$ and PFS $(n=$ 1436). The higher the expression of MED29 was, the worse OS $(P=0.0011)$ (Fig. $2 C)$ and PFS $(P=1.5 e-$ 07) (Fig. 2D) were in patients with OvCa.

\subsection{MED29 is highly expressed in OvCa cell lines}

To investigate the function of MED29, a series of RNA interference experiments were carried out to silence MED29 expression in OvCa cell lines (OVCAR3, ES-2, A2780) using two siRNA sequences targeting MED29. Then, the level of MED29 expression was measured in the above cells under standard culture conditions, and the relative fold change in MED29 expression was determined via RT-qPCR. Compared with cells transfected with siRNA-NC, OVCAR3 and A2780 cells transfected with siRNA-295 and siRNA426 exhibited significantly decreased expression of MED29 (Fig. 3A, 3E) $(P<0.05$ or $P<0.01)$. However, the expression of MED29 was not significantly changed in ES-2 cells between the siRNA-NC-transfected and either the siRNA-295- or siRNA-426-transfected cells (Fig. 3C). MED29 expression was also evaluated at the protein level. Compared with the siRNA-NC-transfected cells, siRNA-295- and siRNA-426-transfected OVCAR3 and A2780 cells showed the signal corresponding to MED29 protein was strongly decreased (Fig. 3B, 3F), while no obvious changes were observed between ES-2 cells transfected with the two MED29-targeted siRNAs and those transfected with siRNA-NC (Fig. 3D). These results were consistent with the RT-qPCR data. MED29 expression is very weak in ES-2 cells and high in OVCAR3 and A2780 cell lines. Therefore, in subsequent experiment, we used OVCAR3 and A2780 cells.

\subsection{MED29 promoted cell proliferation in OvCa cell lines}

Cancer is characterized by enhanced cell proliferation. To determine the effect of MED29 on the proliferative capacities of our cell models, the clonogenicity tests was performed first. The enhancement of clonality is a specific feature of cancer cells. After 14 days of standard culture conditions, the number of colonies formed in OVCAR3 and A2780 cells with siRNA-mediated knockdown of MED29 was greatly reduced compared to that in cells transfected with siRNA-NC $(P<0.05$ or $P<0.01)$ (Fig. 4A-B).

Next, cell proliferation was detected by the EdU assay, an immunochemical detection means to measure the incorporation of a nucleotide analogue into newly copied DNA. The percentage of EdU-positive cells was lower than OVCAR3 and A2780 cells transfected with the two MED29-targeted siRNAs than in cells transfected with siRNA-NC ( $<0.05$ or $\mathrm{P}<0.01)$ (Fig. 4C-F). Furthermore, cell proliferation was tested with 
a CCK8 kit. A total of $5 \times 10^{3}$ cells were initially inoculated, and afterwards the OD value was measured. Consistent with the EdU results, MED29 knockdown with siRNA significantly inhibited cell proliferation ( $P$ $<0.01$ ) (Fig. 4G-H). Therefore, MED29 plays a positive role in controlling cell growth, cell proliferation and colony-forming abilities.

\subsection{MED29 regulated the cell cycle of OVCAR3 and A2780 cells.}

Some studies have shown that in the PANC-1 cell line, silencing the overexpressed complex subunit MED29 can arrest cells in G0/G1 phase, suggesting that MED29 regulates the progression of the cell cycle in tumours [16]. Therefore, in this study, we explored whether MED29 regulates the cell cycle progression of OvCa. Based on the GSEA strategy, the results of KEGG pathway analysis indicated that MED29 was indeed involved in the regulation of the cell cycle (Fig. 5).

Then, MED29 was silenced to identify the specific phase in which the OvCa lines were arrested. After treatment of OVCAR3 and A2780 cells with two siRNAs-MED29 for $48 \mathrm{~h}$, using the flow cytometry according to the procedure of $\mathrm{PI} / \mathrm{RNase}$ sustaining buffer kit (BD PharmingenTM) to detect the changes in the cell cycle distribution. The results are presented in Fig. $6 \mathrm{~A}-\mathrm{B}$, and the percentages of cells in $\mathrm{G} 1, \mathrm{~S}$ and G2/M phase in the control and experimental groups are shown. In OVCAR3 cells, the percentage of cells in $\mathrm{G} 2 / \mathrm{M}$ phase was $16.82 \%$ in the control group, $31.27 \%$ in the siRNA-295 group, and $31.36 \%$ in the siRNA-426 group. Similar to the OVCAR3 cells, in A2780 cells, the percentage of cells in G2/M phase was $17.17 \%$ in the control group, $24.06 \%$ in the siRNA-295 group, and $27.17 \%$ in the siRNA-426 group. Compared with the control group, the experimental groups showed significant increases in the percentage of cells in G2/M phase. Further analysis of cell-cycle-related proteins (Cyclin D1, Cyclin E and Cyclin B1) showed that the signal corresponding to Cyclin B1 was strongly increased while those of Cyclin D1 and Cyclin E were strongly decreased in OVCAR3 and A2780 cells with MED29-mediated siRNA knockdown (Fig. 6C). These results suggest that inhibiting MED29 expression in OvCa could arrest the cell cycle at the $\mathrm{G} 2 / \mathrm{M}$ transition, thus preventing the cell from entering the next phase and consequently affecting cell proliferation.

\subsection{MED29 promoted the migration and invasion of OVCAR3 and A2780 cells}

Migration and invasion are the key steps in tumour progression and metastasis development [17]. Therefore, in vitro Transwell assays were used to study the effect of transient MED29 knockdown on OvCa cell migration and invasion. As shown in Fig. 7A-D, there was significantly decreased migration and invasion of OVCAR3 and A2780 cells with MED29 knockdown compared to cells treated with siRNA-NC. As the epithelial-mesenchymal transition (EMT) process makes an important impact in tumour metastasis, we investigated whether the role of MED29 in cell spreading promoted EMT. As presented in Fig. 7E-F, in OVCAR3 and A2780 cells, MED29 knockdown inhibited fibronectin and N-cadherin expression, but enhanced E-cadherin expression. Thus, these studies suggested that MED29 promoted OvCa cell migration and invasion in vitro. 


\section{Discussion}

The Mediator complex is a highly conserved multisubunit complex required for RNA polymerase II (Pol II)mediated gene transcription in all eukaryotes and acts as a link between transcriptional activators and general transcriptional mechanisms [18-20]. Transcriptional regulation plays an indispensable role in maintaining the stability of the intracellular environment and multiple differentiation and developmental processes [21]. This regulation involves many specialized proteins and protein complexes, all of which need to cooperate with each other to ensure the precise control of the expression of a given gene in a spatiotemporal manner [22]. The current opinion states that mediators are at the core of gene transcription, with different kinase components of mediators regulating the expression of many types of genes [23]. According to its key role in transcriptional regulation, the Mediator complex has been reported to be aberrantly activated in developmental diseases, cancer and metabolic disorders [24-27].

This complex, which including at least 30 polypeptides, can be subdivided into four structurally different submodules: the head, middle, tail, and cyclin-dependent kinase 8 (CDK8) modules (CKM) [28]. MED29, an important component of the Mediator complex, is located in the "head" and is widely expressed in human embryos and adult tissues [29]. The Drosophila melanogaster protein Intersex, an apparent homologue of mammalian MED29, directly interacts with the DNA-binding transcription factor doublesex and coactivates transcription [30]. In fact, mammalian IXL has been considered as a subunit of the Mediator complex which transduces regulatory signals from DNA-binding transcription factors to Pol II, regulating mRNA synthesis [31, 32]. Therefore, mammalian MED29 may also be the target of one or more DNA-binding transcription activators.

Studies have shown that in pancreatic cancer, overexpression of MED29 activates the processes involved in regulating cell growth, and downregulation of MED29 expression significantly reduces the viability of PANC-1 cells, indicating that MED29 has a key role in the development and regulation of pancreatic cancer and is an ideal potential therapeutic target $[16,33]$. Deng et al. found that MED29 is the subunit gene that carries more SCNAs and is overexpressed in OvCa. MED29 is co-amplified with PAF1 and SUPT5H, which encode two critical factors engaged in Pol II extension and interact with the Mediator complex directly [34]. These findings imply an important function of MED29 in the occurrence and development of tumours. However, the mechanism by which MED29 promotes the progression of OvCa remains unknown.

In our study, the data obtained from the TCGA database showed that 19q13.2 AMP was highly relevant poor survival in OvCa patients (Fig. 1A), and MED29 was the most obvious gene amplified in this region (Fig. 1B). In addition, with regard to the effect of 19q13.2 AMP on gene expression in the region, CNA upregulated the expression of the MED29 gene (Fig. 1C). The data from the GSE29450 dataset obtained from GEO showed that the MED29 gene is overexpressed in OvCa. These results were verified by IHC (Fig. 2A-B). In addition, the data obtained from Kaplan-Meier Plotter indicated that the higher the expression of MED29 was, the worse the OS $(p=0.0011)$ and PFS $(p=1.5 e-07)$ in OvCa. 
We established two cells model with MED29 knockdown to explore the role and mechanism of MED29 in OvCa. As shown in Fig. 3, MED29 expression was downregulated after transfection of MED29-specfic siRNAs into two cell lines than the siRNA-NC cells, $\mathrm{OVCAR}_{3}$ and $A 2780$ cells transfected with two siRNAs showed dramatic inhibition of proliferation (Fig. 4), suggesting that MED29 promotes cell proliferation in OvCa. Data analysis based on the KEGG pathway showed that MED29 might be closely related to the cell cycle (Fig. 5). Studies have shown that in the PANC-1 pancreatic cancer cell line, silencing the overexpressed complex subunit MED29 can arrest cells in G0/G1 phase, suggesting that MED29 regulates the cell cycle progression of tumour cells [18]. Therefore, the effect of silencing MED29 on the regulation of the cell cycle in OvCa cell lines was tested. As shown in Fig. 6, silencing MED29 caused OvCa cells to arrest at $\mathrm{G} 2 / \mathrm{M}$ transition, and the expression of cycle-related proteins was also affected, indicating that MED 29 affects this regulatory mechanism in cells. Since EMT plays an indispensable role in the process of tumour metastasis, the role of MED29 in promoting EMT was investigated. As shown in Fig. 7, in OVCAR3 and A2780 cells, MED29 knockdown inhibited cell migration and invasion. When taken into account with previous research results, our data serve as the groundwork for more experiments to study the role of MED29 in regulating the occurrence and development of OvCa; furthermore, MED29 may be a target for OvCa therapies.

In conclusion, MED29 is highly expressed in OvCa tissues compared with non-tumour tissues. Analysis showed that OvCa patients with high MED29 expression have poor prognosis. The cell proliferation results suggested that MED29 plays a critical role in OvCa cell proliferation. In addition, MED29 participates in the regulation of the OvCa cell cycle and promotes cell migration and invasion. Our results indicate that MED29 might be a novel diagnostic marker and therapeutic target of OvCa.

\section{Declarations}

\section{Ethics approval and consent to participate}

Not applicable

\section{Consent for publication}

Not applicable.

\section{Availability of data and materials}

The datasets used and/or analyzed during the current study are available from the publicly available TCGA and GEO databases

\section{Competing interests}

The authors declare that they have no competing interests

\section{Funding}


National Natural Science Foundation of China, Grant/Award Number: \#81760504; the Natural Science Foundation of Jiangxi Province Grant/Award Number: \#S2016ABC20022.

\section{Authors' contributions}

HW and FF designed the study, HW and ML participated in data selection and assembly. LC,GW and HN performed the data analysis. HW, LC and GW and were involved in drafting the manuscript. FF and LT revised the manuscript critically for important intellectual content. All authors read and approved the final manuscript.

\section{Acknowledgments}

This work was supported by the National Natural Science Foundation of China (Grant No. \#81760504 to F Fu) and the Natural Science Foundation of Jiangxi Province (Grant No. \#20161ACB20022 to to F. Fu).

\section{References}

1. Ferlay J, Shin HR, Bray F, Forman D, Mathers C, Parkin DM. Estimates of worldwide burden of cancer in 2008: GLOBOCAN 2008. 2010;127(12):2893-917.

2. Lengyel E. Ovarian cancer development metastasis. 2010;177(3):1053-64.

3. Siegel RL, Miller KD, Jemal A. Cancer statistics, 2016. CA Cancer J Clin. 2016;66(1):7-30.

4. Webb PM, Jordan SJ. Epidemiology of epithelial ovarian cancer. Best Pract Res Clin Obstet Gynaecol. 2017;41:3-14.

5. Albertson DG, Collins C, McCormick F, Gray JW. Chromosome aberrations in solid tumors. Nat Genet. 2003;34(4):369-76.

6. Beroukhim R, Mermel CH, Porter D, Wei G, Raychaudhuri S, Donovan J, et al. The landscape of somatic copy-number alteration across human cancers. Nature. 2010;463(7283):899-905.

7. Curtis C, Shah SP, Chin SF, Turashvili G, Rueda OM, Dunning MJ, et al. The genomic and transcriptomic architecture of 2,000 breast tumours reveals novel subgroups. Nature. 2012;486(7403):346-52.

8. Futreal PA, Coin L, Marshall M, Down T, Hubbard T, Wooster R, et al. A census of human cancer genes. Nat Rev Cancer. 2004;4(3):177-83.

9. Zarrei M, MacDonald JR, Merico D, Scherer SW. A copy number variation map of the human genome. Nat Rev Genet. 2015;16(3):172-83.

10. Lin DC, Wang MR, Koeffler HP. Genomic and Epigenomic Aberrations in Esophageal Squamous Cell Carcinoma and Implications for Patients. Gastroenterology. 2018;154(2):374-89.

11. Patch AM, Christie EL, Etemadmoghadam D, Garsed DW, George J, Fereday S, et al. Whole-genome characterization of chemoresistant ovarian cancer. Nature. 2015;521(7553):489-94.

12. Integrated genomic analyses of ovarian carcinoma. Nature. 2011;474(7353):609-15. 
13. Kelemen LE, Lawrenson K, Tyrer J, Li Q, Lee JM, Seo JH, et al. Genome-wide significant risk associations for mucinous ovarian carcinoma. Nat Genet. 2015;47(8):888-97.

14. Edgar R, Domrachev M, Lash AE. Gene Expression Omnibus: NCBI gene expression and hybridization array data repository. Nucleic Acids Res. 2002;30(1):207-10.

15. Varghese F, Bukhari AB, Malhotra R, De A. IHC Profiler: an open source plugin for the quantitative evaluation and automated scoring of immunohistochemistry images of human tissue samples. PLoS One. 2014;9(5):e96801.

16. Kuuselo R, Savinainen K, Sandström S, Autio R, Kallioniemi A. MED29, a component of the mediator complex, possesses both oncogenic and tumor suppressive characteristics in pancreatic cancer. 2011. 129(11): p. 2553-2565.

17. Fidler IJ. Understanding bone metastases: the key to the effective treatment of prostate cancer. Clin Adv Hematol Oncol. 2003;1(5):278-9.

18. Ansari SA, He Q, Morse RH. Mediator complex association with constitutively transcribed genes in yeast. Proceedings of the National Academy of Sciences, 2009. 106(39): p. 16734-16739.

19. Kornberg RD. Mediator and the mechanism of transcriptional activation. Trends Biochem Sci. 2005;30(5):235-9.

20. Allen BL, Taatjes DJ. The Mediator complex: a central integrator of transcription. Nat Rev Mol Cell Biol. 2015;16(3):155-66.

21. Koch F, Jourquin F, Ferrier P, Andrau JC. Genome-wide RNA polymerase II: not genes only! Trends Biochem Sci. 2008;33(6):265-73.

22. Taatjes DJ, Marr MT, Tjian R. Regulatory diversity among metazoan co-activator complexes. Nat Rev Mol Cell Biol. 2004;5(5):403-10.

23. Poss ZC, Ebmeier CC, Taatjes DJ. The Mediator complex and transcription regulation. Crit Rev Biochem Mol Biol. 2013;48(6):575-608.

24. Spaeth JM, Kim NH, Boyer TG. Mediator and human disease. Semin Cell Dev Biol. 2011;22(7):77687.

25. Xiaoli, Yang F. Mediating lipid biosynthesis: implications for cardiovascular disease. Trends Cardiovasc Med. 2013;23(7):269-73.

26. Shaikhibrahim Z, Menon R, Braun M, Offermann A, Queisser A, Boehm D, et al. MED15, encoding a subunit of the mediator complex, is overexpressed at high frequency in castration-resistant prostate cancer. 2014. 135(1): p. 19-26.

27. Jin F, Irshad S, Yu W, Belakavadi M, Chekmareva M, Ittmann MM, et al. ERK and AKT signaling drive MED1 overexpression in prostate cancer in association with elevated proliferation and tumorigenicity. Mol Cancer Res. 2013;11(7):736-47.

28. Yin JW, Wang G. The Mediator complex: a master coordinator of transcription and cell lineage development. Development. 2014;141(5):977-87. 
29. Conaway RC, Sato S, Tomomori-Sato C, Yao T, Conaway JW. The mammalian Mediator complex and its role in transcriptional regulation. Trends in biochemical sciences; 2005.

30. (5): p. 250-255. 30. Garrett-Engele CM, Siegal ML, Manoli DS, Williams BC, Li H, Baker BS. intersex, a gene required for female sexual development in Drosophila, is expressed in both sexes and functions together with doublesex to regulate terminal differentiation. Development. 2002. 129(20): p. 46614675.

31. Wang Y, Li Y, Zeng W, Zhu C, Xiao J, Yuan W, et al. IXL, a new subunit of the mammalian mediator complex, functions as a transcriptional suppressor. Biochem Biophys Res Commun. 2004;325(4):1330-8.

32. Sato S, Tomomori-Sato C, Banks CA, Parmely TJ, Sorokina I, Brower CS, et al. A mammalian homolog of Drosophila melanogaster transcriptional coactivator intersex is a subunit of the mammalian Mediator complex. J Biol Chem. 2003;278(50):49671-4.

33. Chen S, Auletta T, Dovirak O, Hutter C, Kuntz K, El-ftesi S, et al. Mediator complex components are frequent targets for genetic alterations in various types of human cancer. J Genet Genomics. 2017;44(12):587-91.

34. Deng $L$, et al. Mediator complex components are frequent targets for genetic alterations in various types of human cancer. J Genet Genomics. 2017;44(12):587-91.

\section{Figures}


A

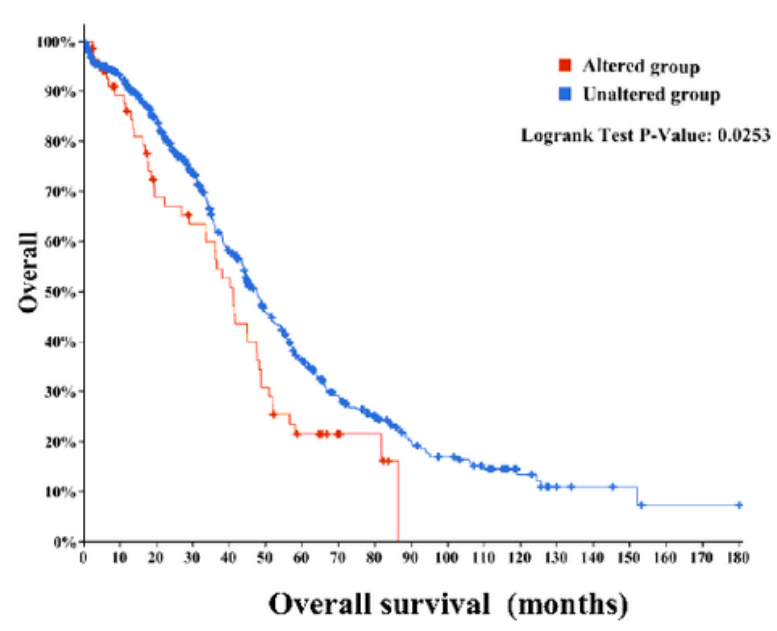

C

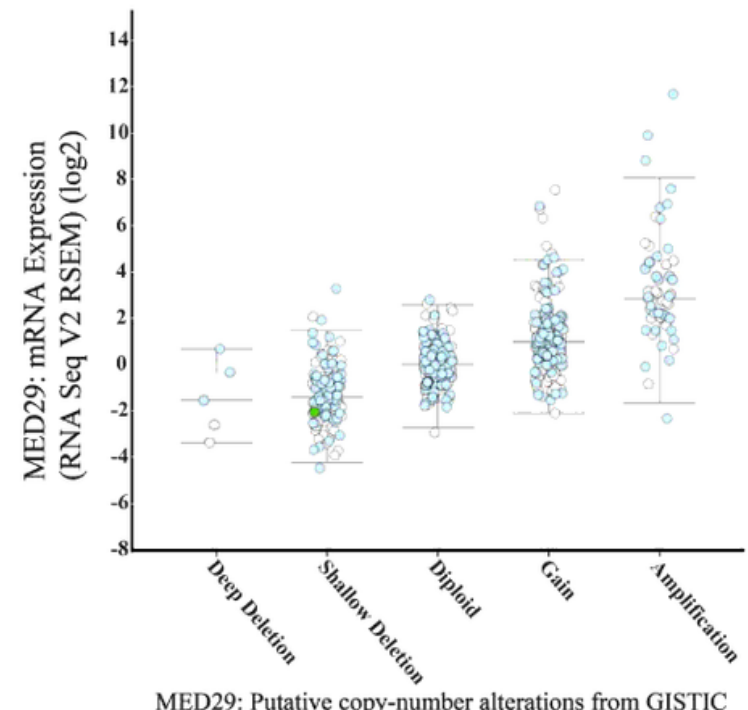

- Missense (VUS) Not mutated Not profiled for mutations

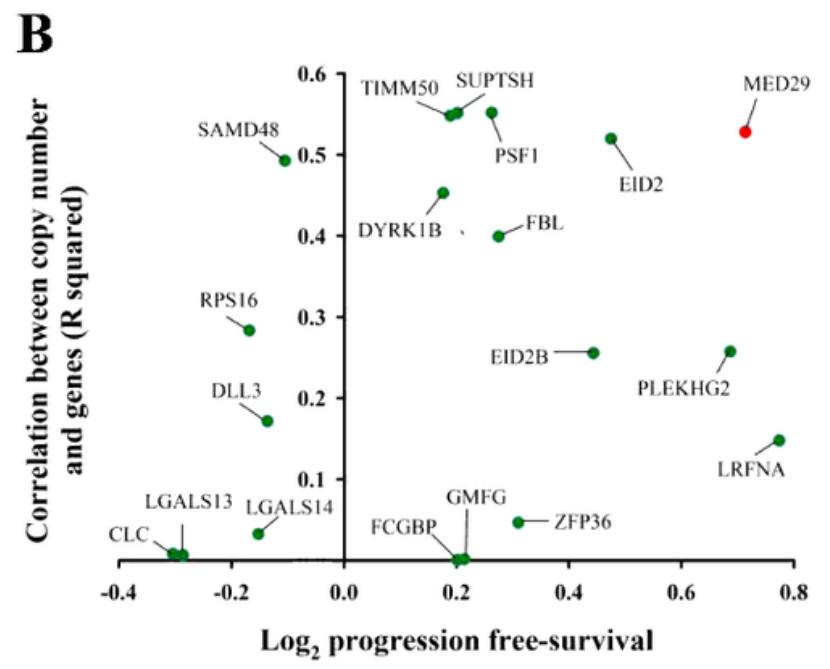

D

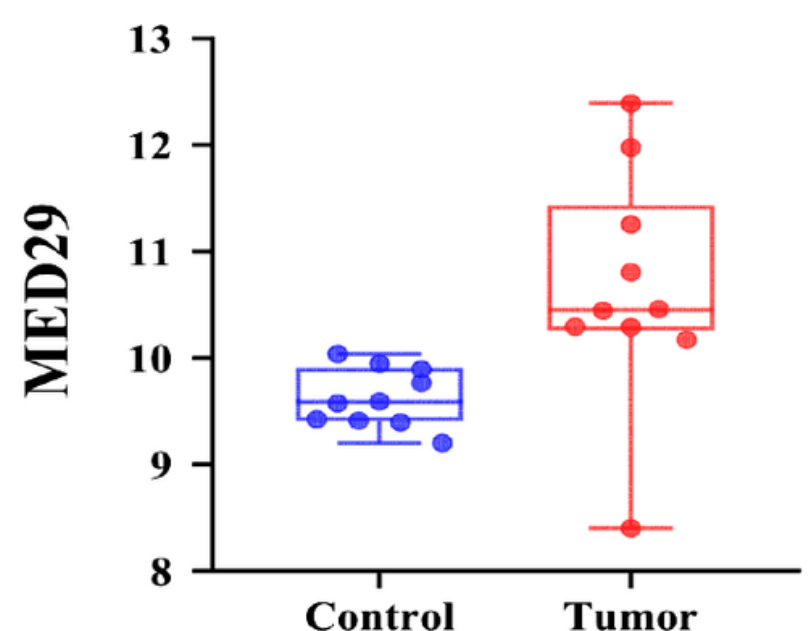

\section{Figure 1}

MED29 expression was most obviously changed in patients with 19q13.2 AMP. A. The 19q13.2 AMP was significantly associated with poor survival in OvCa patients. B. The AMP of the MED29 has the greatest impact on the progression free survival of patients. C. The 19q13.2 AMP increased the MED29 mRNA level. D. expression of MED29 in the OvCa samples were significantly higher than the normal samples (P $=0.0015)$. 
A

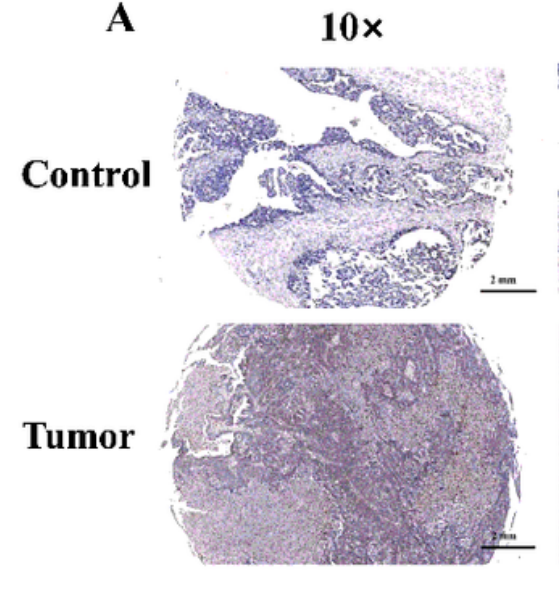

C

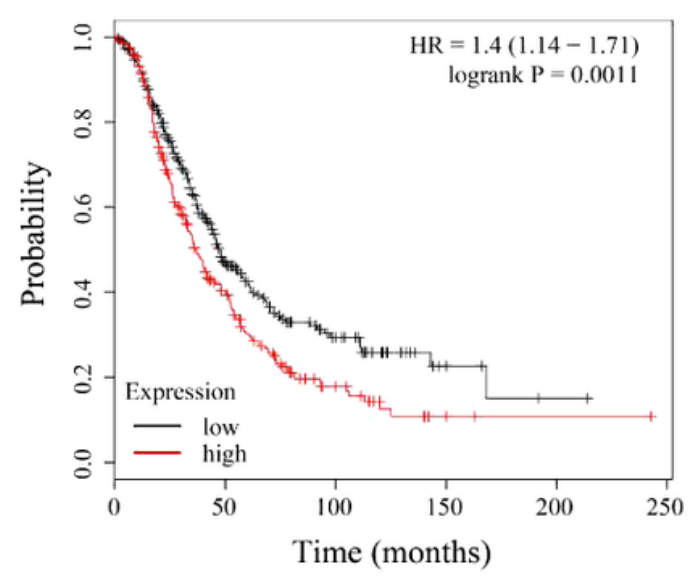

$40 \times$
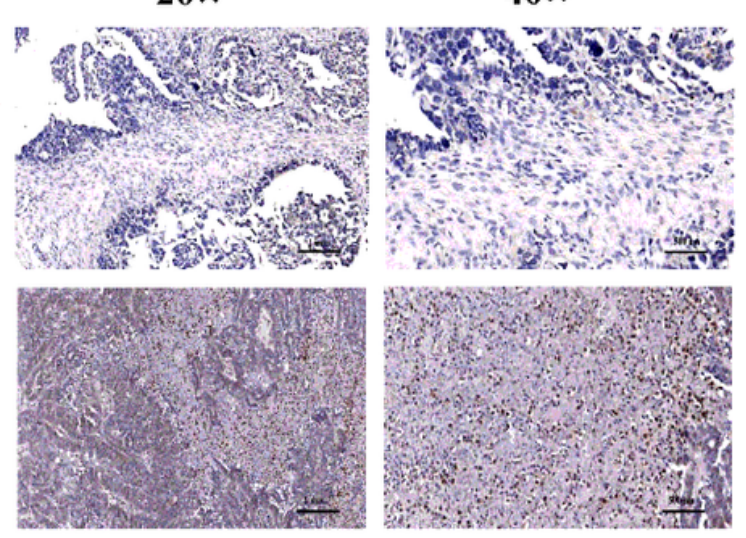

B

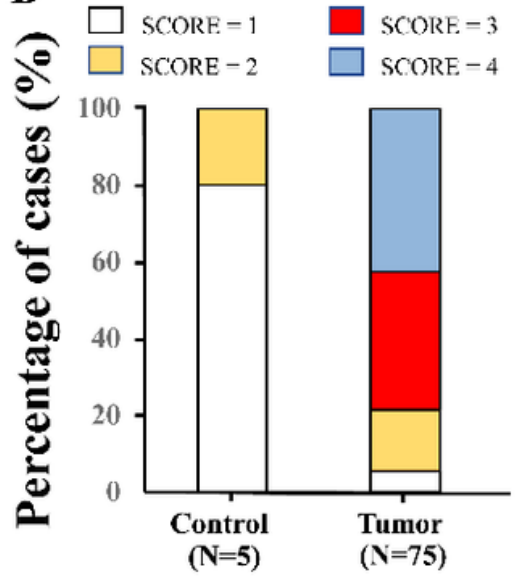

D

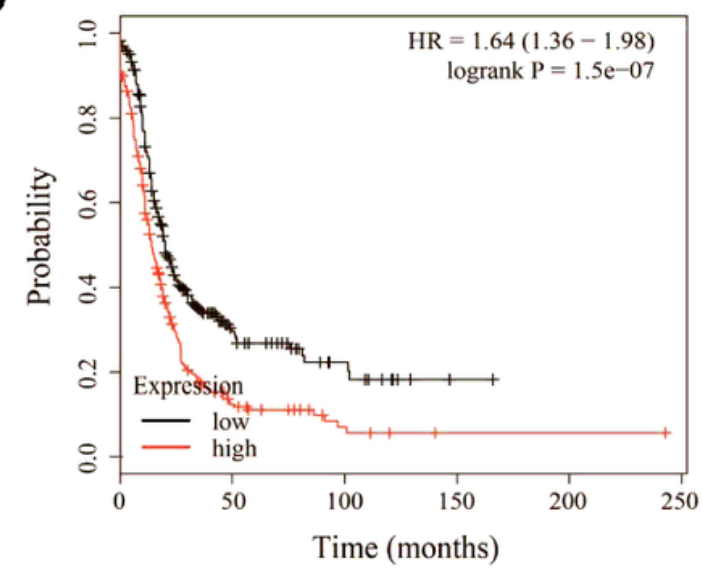

Figure 2

MED29 is highly expressed in OvCa and is related to poor prognosis of OvCa. A. MED29 stains stronger in OvCa tissues found through IHC analysis. B. Scoring 80 cases of ovarian tissues (5 cases of normal tissues and 75 cases of OvCa tissues) by ImageJ. C. The relationship between the expression of MED29 and OS in patients with OvCa. D. The relationship between the expression of MED29 and PFS in patients with OvCa. 

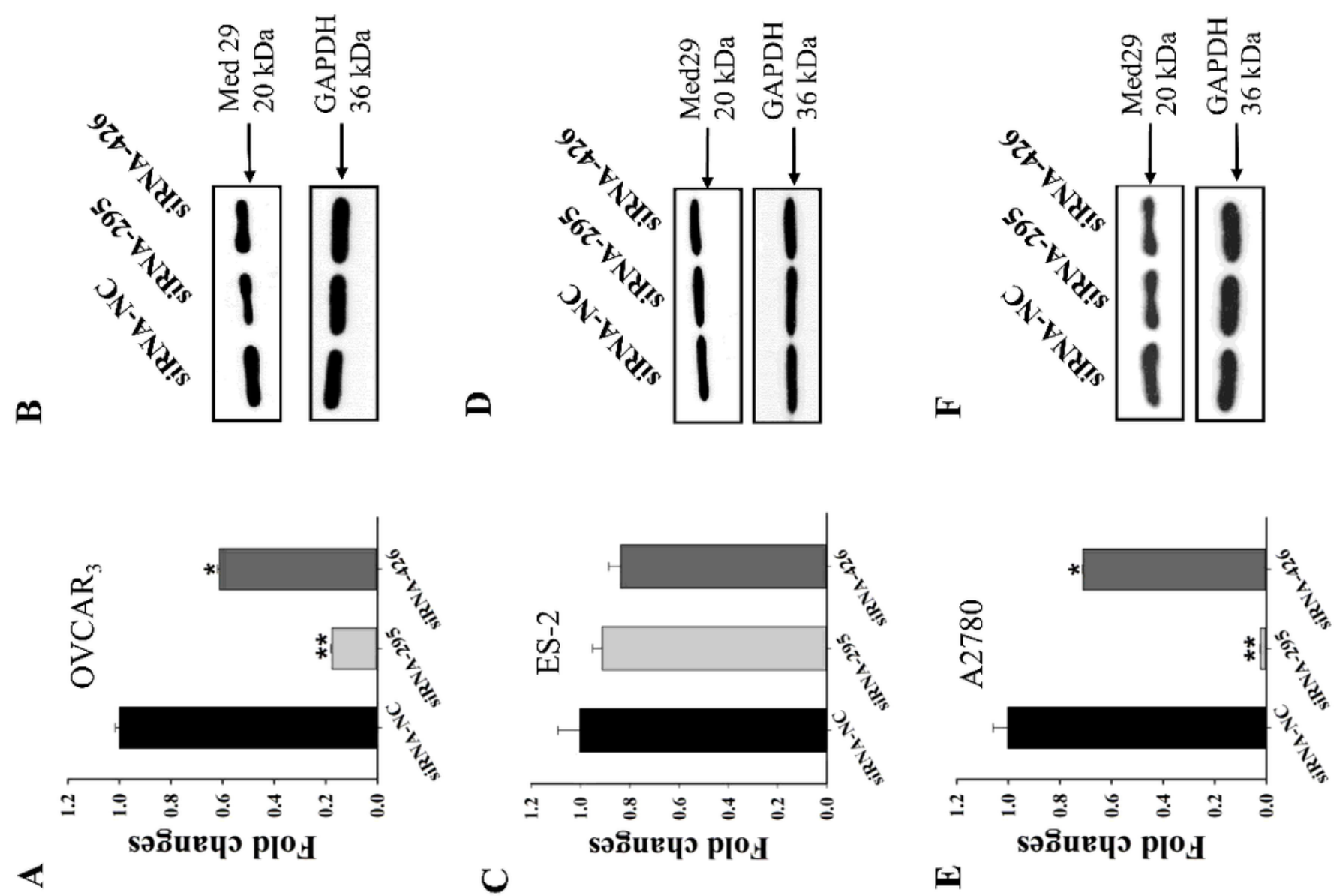

Figure 3

MED29 is highly expressed in OVCAR3 and A2780 cells. A. Relative expression of MED29 mRNA in OVCAR3 cells. B. Western blot analysis of MED29 expression in OVCAR3 cells. C. Relative expression of MED29 mRNA in ES-2 cells. D. Western blot analysis of MED29 expression in ES-2 cells. E. Relative expression of MED29 mRNA in A2780 cells. F. Western blot analysis of MED29 expression in A2780 cells. Data are means $\pm S D$ of three independent experiments. ${ }^{*} P<0.05$, ${ }^{*} P<0.01$, compared to control. Total proteins $(25 \mathrm{ug}$ ) were separated on SDS-PAGE and transferred to PVDF membranes. Proteins were detected using anti-MED29 and anti-GAPDH antibodies. 
A

SiRNA-NC

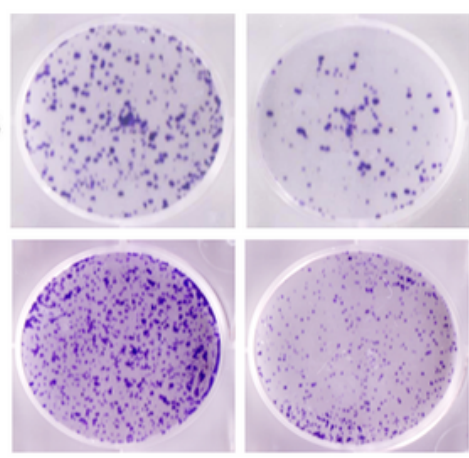

$\mathbf{A 2 7 8 0}$

OVCAR3

C

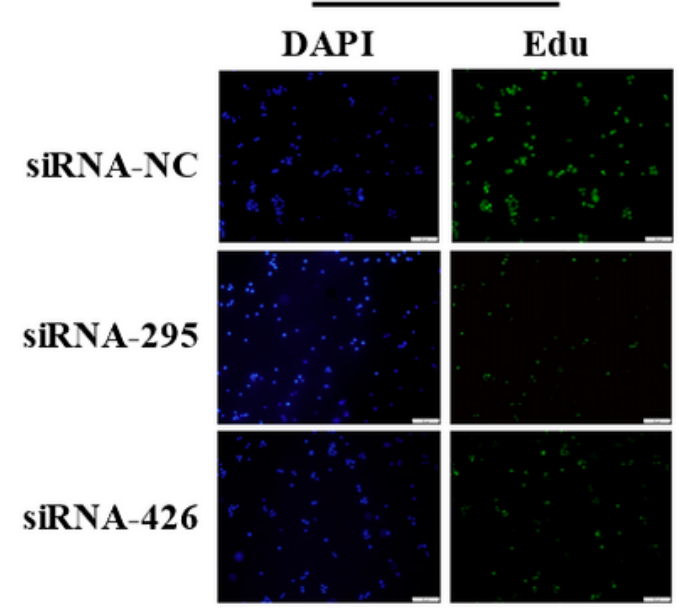

E

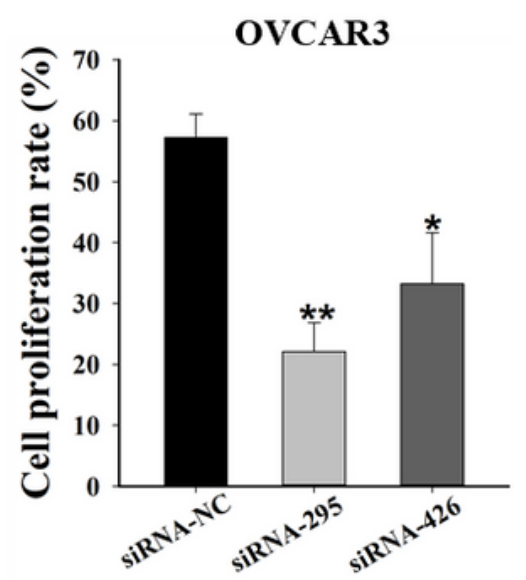

siRNA-426
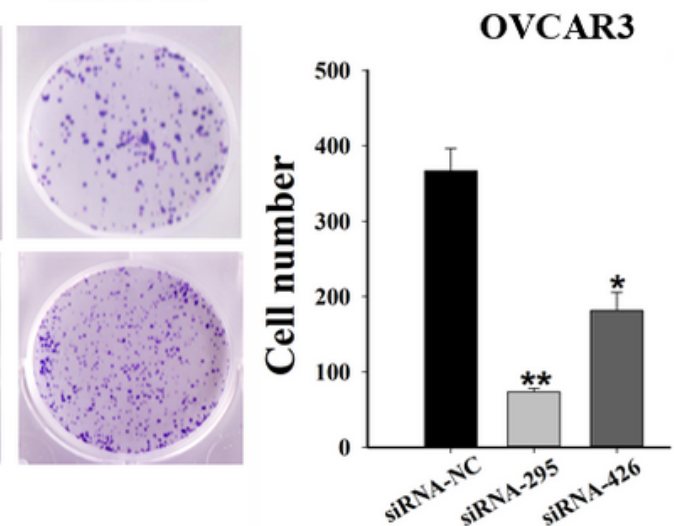

$\mathbf{A 2 7 8 0}$

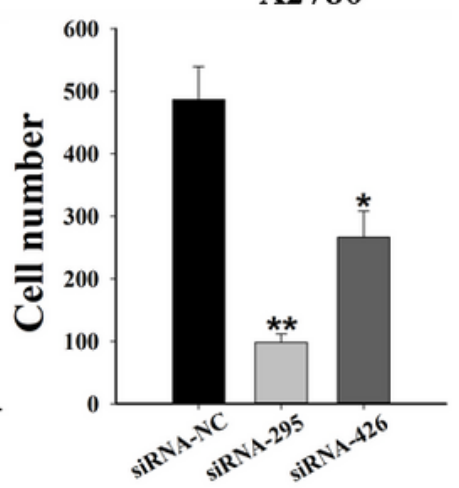

G

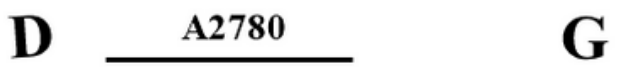

DAPI Edu
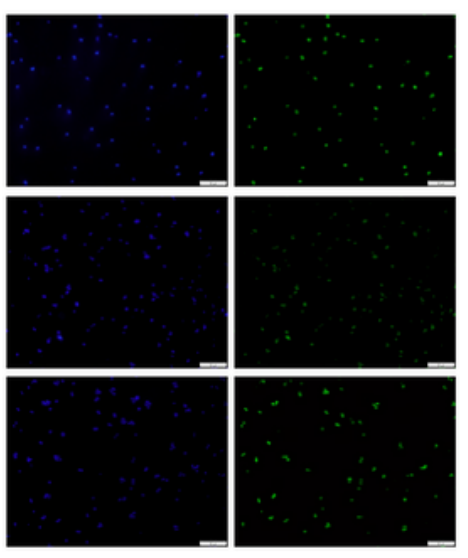

F

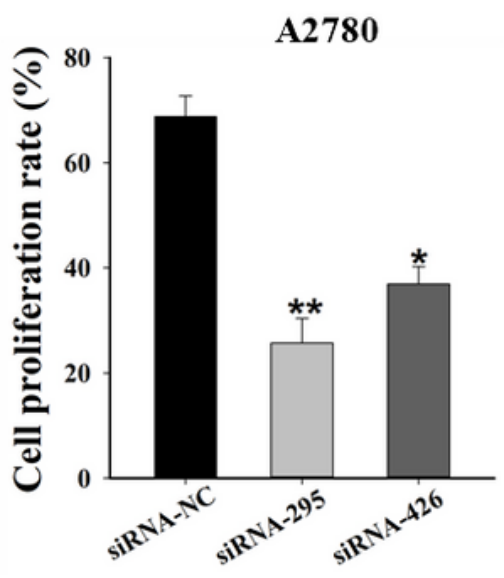

OVCAR3

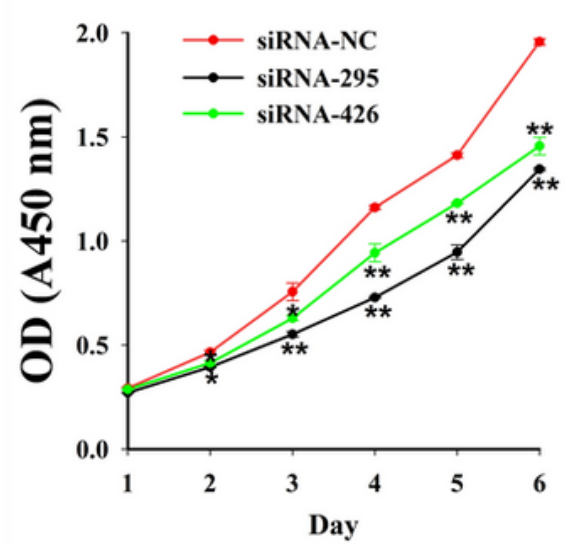

H

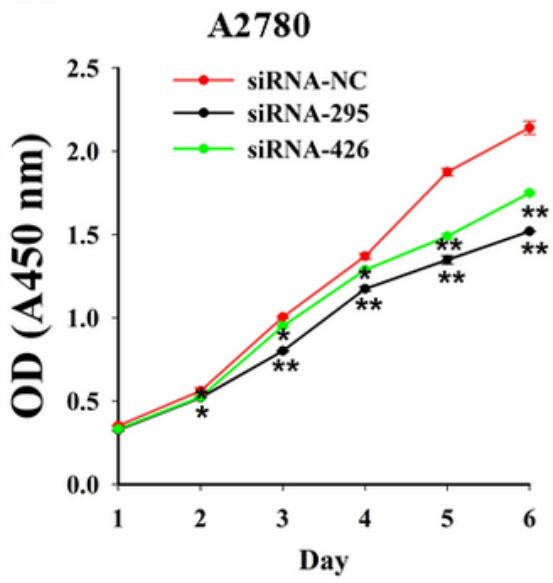

Figure 4

MED29 promoted cell proliferation in OvCa cells. A. Effect of MED29 on colony formation in OVCAR3 and A2780 cells. B. Quantitative changes in OVCAR3 and A2780 colony formation were evaluated by counting using ImageJ. C. The positive rate of cell proliferation of OVCAR3 cells by Edu experiment. D. The positive rate of cell proliferation of A2780 cells by Edu experiment. E. Cell proliferation rate in OVCAR3 cells was calculated by ImageJ. F. Cell proliferation rate in A2780 cells was calculated by ImageJ. G. Proliferation 
of OVCAR3 cells with the CCK8 kit. H. Proliferation of A2780 cells with the CCK8 kit. Results shown are representative of three independent experiments. Values were normalized to control cells and represent mean \pm SD of quintuplicate determinations. Student's $t$ test was used for comparisons ( $P \leq 0.05$, ** $P \leq 0.01)$.

A

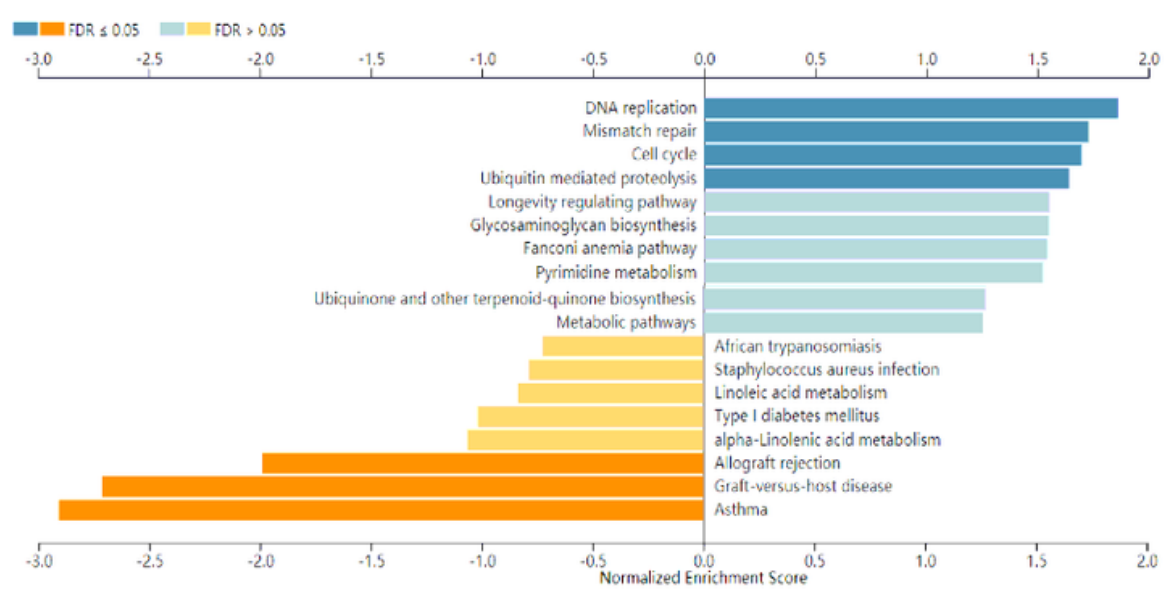

B

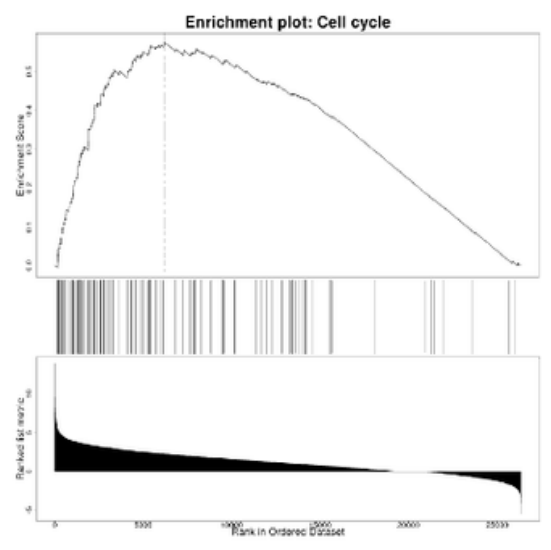

\section{Figure 5}

MED29 was involved in the regulation of cell cycle progression based on pathway enrichment analysis. A. KEGG pathway enrichment analysis of genes positively and negatively associated with MED29. B. MED29 is associated with cell cycle. 


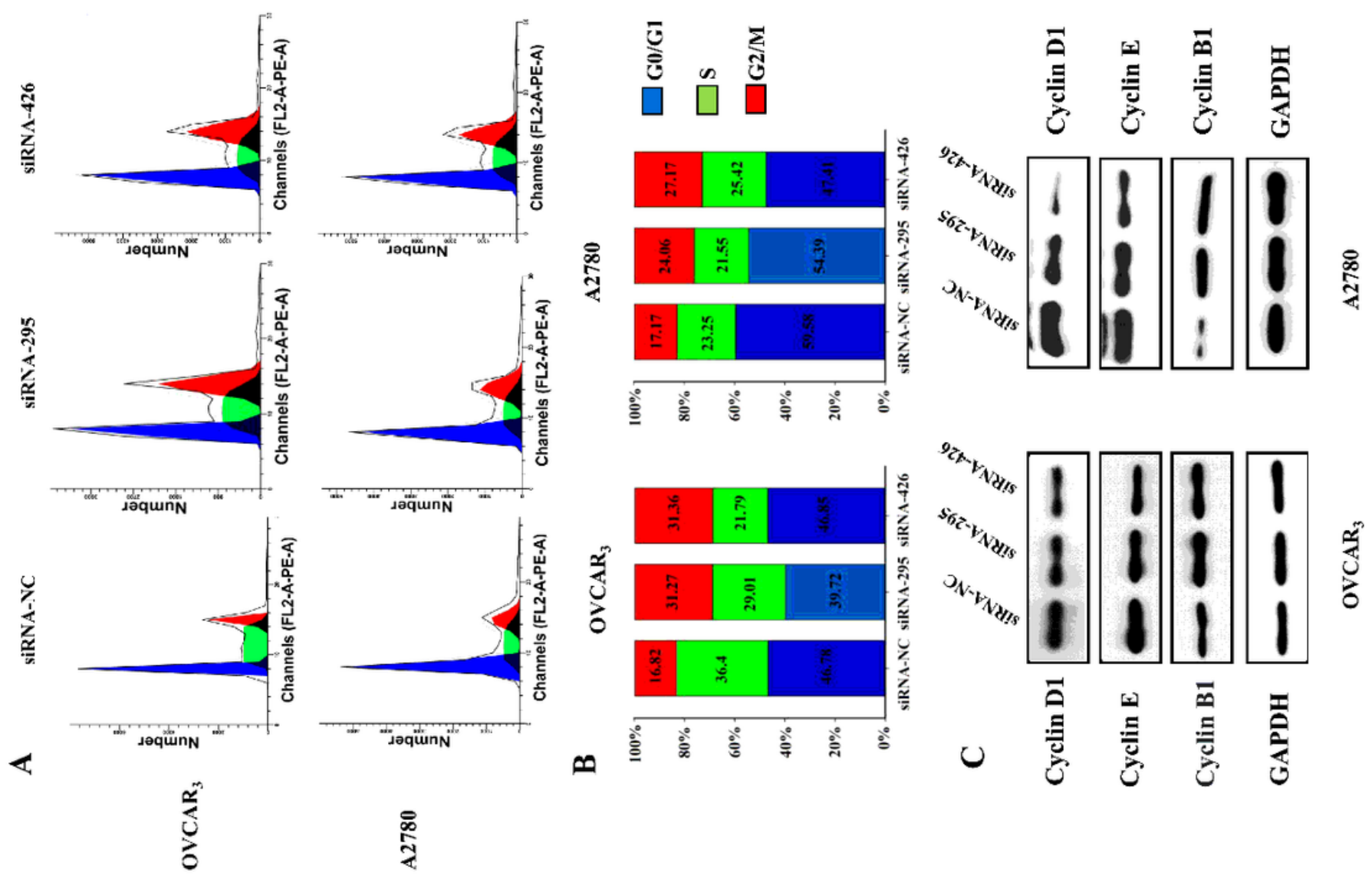

Figure 6

MED29 regulated the cell cycle progression of OVCAR3 and A2780 cells. A. Cell cycle detection of OVCAR3 and A2780 cells by flow cytometry. B. Percentage of OVCAR3 and A2780 cells in each phase of the cell cycle. C. Western blot analysis of cell cycle related proteins in OVCAR3 cells and A2780 cells silenced by MED29. Total proteins ( $25 \mathrm{ug}$ ) were separated on SDS-PAGE and transferred to PVDF membranes. Proteins were detected using anti-CyclinD1, anti-CyclinE, anti-CyclinB1 and anti-GAPDH antibodies. Results shown are representative of three independent experiments. 


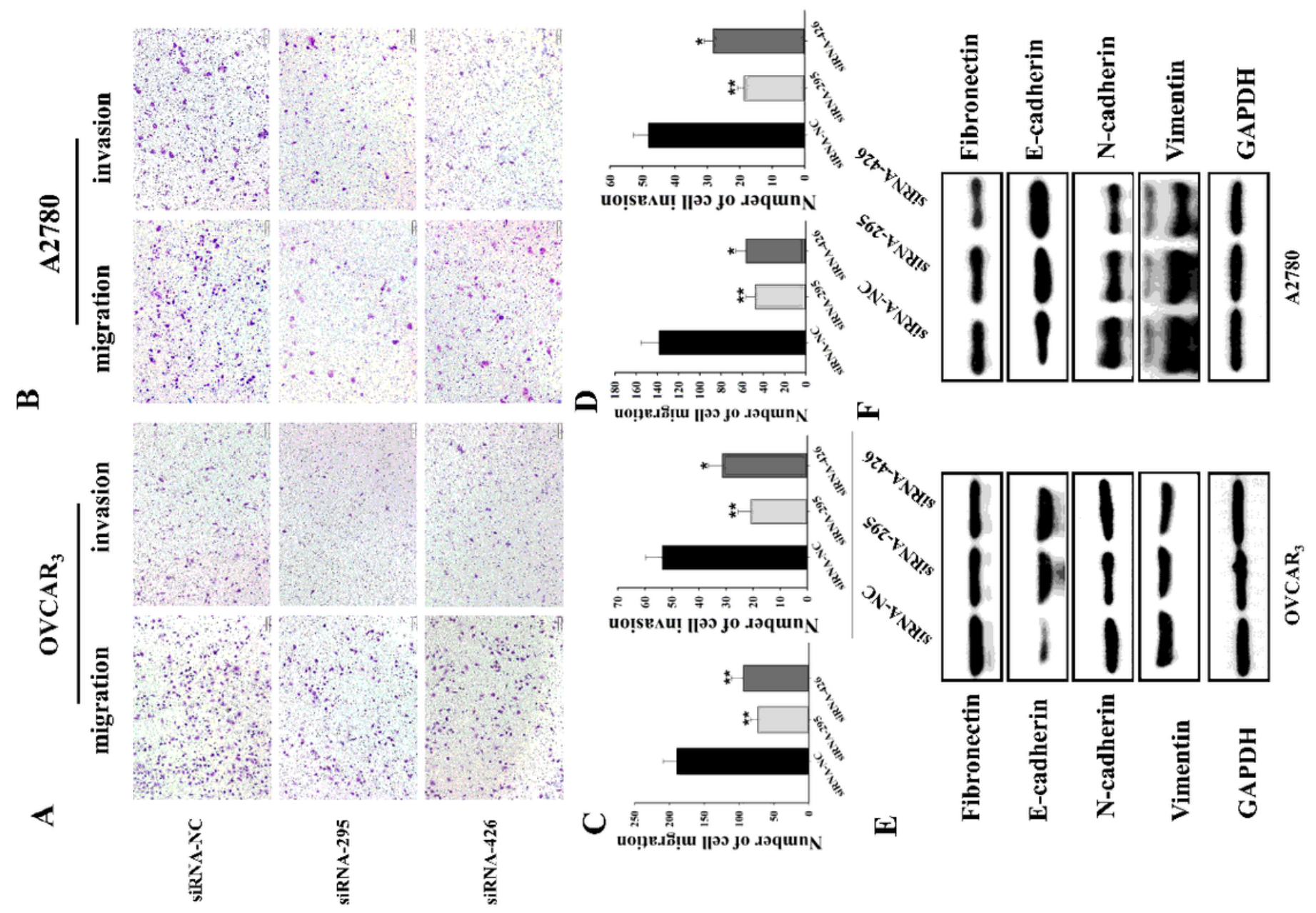

Figure 7

MED29 promoted cell migration and invasion in OvCa cells. A. The cell migration and invasion in OVCAR3 cells. B. The cell migration and invasion in A2780 cells. C. Quantitative analysis of OVCAR3 cell migration and invasion by ImageJ. D. Quantitative analysis of A2780 cell migration and invasion by ImageJ. Migration and invasion assays were performed in MED29 knockdown OVCAR3 and A2780 cells transfected with siRNAs and negative controls. E. Western blot analysis of EMT related proteins in OVCAR3 cells silenced by MED29. F. Western blot analysis of EMT related proteins in A2780 cells silenced by MED29. Total proteins ( $25 \mathrm{ug}$ ) were separated on SDS-PAGE and transferred to PVDF membranes. Proteins were detected using anti-Fibronectin, anti-E-cadherin, anti-N-cadherin, anti-Vimentin and anti-GAPDH antibodies. Values were normalized to control cells and represent mean \pm SD of quintuplicate determinations. Student's $t$ test was used for comparisons $\left({ }^{\star} P \leq 0.05,{ }^{\star} \mathrm{P} \leq 0.01\right)$. 